\title{
BMJ
}

\section{Efficacy of statins in familial hypercholesterolaemia: a long term cohort study}

Jorie Versmissen, researcher, ${ }^{1}$ Daniëlla M Oosterveer, researcher, ${ }^{1}$ Mojgan Yazdanpanah, epidemiologist, ${ }^{1}$ Joep C Defesche, senior researcher, ${ }^{2}$ Dick C G Basart, clinician, ${ }^{3}$ Anho H Liem, clinician, ${ }^{4}$ Jan Heeringa, statistician, ${ }^{5}$ Jacqueline C Witteman, professor of epidemiology, ${ }^{5}$ Peter J Lansberg, clinician, ${ }^{2}$ John J P Kastelein, professor of vascular medicine, ${ }^{2}$ Eric J G Sijbrands, associate professor ${ }^{1}$

$\overline{{ }^{1} \text { Department of Internal Medicine, }}$ Erasmus University Medical Centre, PO box 2040, 3000 CA Rotterdam, Netherlands

${ }^{2}$ Department of Vascular Medicine, Academic Medical Centre, PO box 22660, 1100 DD Amsterdam, Netherlands ${ }^{3}$ Department of Cardiology, Westfries Gasthuis, PO box 600, 1620 AR Hoorn, Netherlands

${ }^{4}$ Department of Cardiology, Oosterschelde Hospital, PO box 106, 4460 BB Goes, Netherlands

${ }^{5}$ Department of Epidemiology and Biostatistics, Erasmus University Medical Centre, Rotterdam Correspondence to: EJ G Sijbrands e.sijbrands@erasmusmc.nl

Cite this as: $B M J$ 2008;337:a2423 doi:10.1136/bmj.a2423

\section{ABSTRACT}

Objective To determine the efficacy of statin treatment on risk of coronary heart disease in patients with familial hypercholesterolaemia.

Design Cohort study with a mean follow-up of 8.5 years. Setting 27 outpatient lipid clinics.

Subjects 2146 patients with familial

hypercholesterolaemia without prevalent coronary heart disease before 1 January 1990.

Main outcome measures Risk of coronary heart disease in treated and "untreated" (delay in starting statin treatment) patients compared with a Cox regression model in which statin use was a time dependent variable.

Results In January 1990, 413 (21\%) of the patients had started statin treatment, and during follow-up another 1294 patients $(66 \%)$ started after a mean delay of 4.3 years. Most patients received simvastatin $(n=1167$, $33 \mathrm{mg}$ daily) or atorvastatin ( $n=211,49 \mathrm{mg}$ daily). We observed an overall risk reduction of $76 \%$ (hazard ratio 0.24 (95\% confidence interval 0.18 to 0.30 ), P<0.001). In fact, the risk of myocardial infarction in these statin treated patients was not significantly greater than that in an age-matched sample from the general population (hazard ration 1.44 (0.80 to 2.60), $\mathrm{P}=0.23$ ).

Conclusion Lower statin doses than those currently advised reduced the risk of coronary heart disease to a greater extent than anticipated in patients with familial hypercholesterolaemia. With statin treatment, such patients no longer have a risk of myocardial infarction significantly different from that of the general population.

\section{INTRODUCTION}

Familial hypercholesterolaemia is a monogenic disorder associated with a greatly increased risk of coronary heart disease. ${ }^{1}$ Statins are first choice treatment for all patients with the condition. ${ }^{2}$ Placebo controlled trials were not carried out in these patients when statins were introduced, as it was considered unethical to withhold causal treatment from patients with an inborn error of lipid metabolism. ${ }^{3}$ We therefore lack estimates of the true efficacy of statin treatment in such patients. The results of the atorvastatin versus simvastatin on atherosclerotic progression (ASAP) study have suggested that we should treat such patients preferably with potent statins to maximally decrease low density lipoprotein (LDL) cholesterol concentrations and induce regression of atherosclerosis. ${ }^{45}$ In the recently published ENHANCE study, adding ezetimibe to the highest dose of simvastatin resulted in substantial further reductions of LDL cholesterol concentrations and high sensitivity $\mathrm{C}$ reactive protein levels but did not further reduce the carotid intimamedia thickness. ${ }^{6}$ One possible explanation for these results is that patients with familial hypercholesterolaemia are currently treated optimally with statins, resulting in delipidated arterial walls of normal thickness, and, therefore, further lowering of LDL cholesterol levels has no beneficial effect. In two observational studies, secular trends suggest that statins have roughly halved the risk of coronary heart disease in patients with familial hypercholesterolaemia. ${ }^{78}$ However, the exact prognosis of treated asymptomatic patients remains unknown, and this lack of hard endpoint data has, for example, limited access to life insurance. $^{9}$

In this study we investigated the effect of statins on the risk of incident coronary heart disease in patients with familial hypercholesterolaemia, using the variation in the time of starting statin treatment to mimic a clinical trial.

\section{METHODS}

\section{Study population}

During 1989-2002, we recruited a cohort of 2400 patients with familial hypercholesterolaemia from 27 lipid clinics as described previously. ${ }^{10}$ We reviewed medical records to establish extensive phenotypic data including anthropometric measures, cardiovascular events, and use of lipid lowering drugs. ${ }^{11}$

In the present study, we chose 1 January 1990, just after the first statin (simvastatin) became available in the Netherlands, as the start point. We excluded patients who already had coronary heart disease by 1990 to mimic a controlled primary prevention trial starting at the introduction of the first statin. We determined serum lipid concentrations in fasting 
Patients with familial hypercholesterolaemia $(n=2400)$

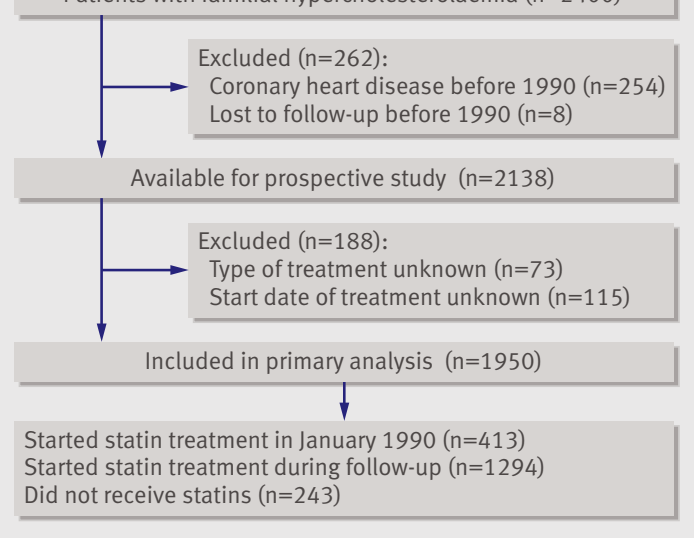

Fig 1 | Flow of patients through study

patients who had not used lipid lowering drugs for at least six weeks, and calculated LDL cholesterol concentrations using the Friedewald formula. ${ }^{12}$

In addition, we compared the risk of incident myocardial infarction in patients with familial hypercholesterolaemia with that in the general population. For this analysis, we selected patients with familial hypercholesterolaemia who were older than 55 years at 1 January 1990 and a selection of the population in the Rotterdam study matched as a group for age and sex. The Rotterdam study is a large population based, prospective, follow-up study starting in 1990 assessing the disease burden in elderly people. ${ }^{13}$

\section{Outcome measures}

We defined coronary heart disease in our study cohort as at least one of the following:

- Myocardial infarction, proved by at least two of the following:

Classic symptoms ( $>15$ minutes)

Specific electrocardiographic abnormalities

Elevated cardiac enzymes $(>2 \times$ upper limit of normal)

- Percutaneous coronary intervention or other invasive procedures

- Coronary artery bypass grafting

- Angina pectoris, diagnosed as classic symptoms in combination with at least one unequivocal result of one of the following:

\section{Exercise test}

Nuclear scintigram

Dobutamine stress ultrasound scan

$>70 \%$ stenosis on a coronary angiogram.

In the Rotterdam study, no data on angina were available. We therefore chose to study myocardial infarction as the end point in this analysis. Patients with percutaneous transluminal coronary angioplasty, coronary bypass grafting, or myocardial infarction were excluded from the sample of patients from our cohort and as from the selected population from the Rotterdam study.

\section{Statistical analysis}

We compared patients' general characteristics using analysis of variance for continuous variables and $\chi^{2}$ test for categorical variables. We analysed lipid concentrations, after adjustment for age and sex, with multiple linear regression analyses. We calculated absolute risk by taking into account whether statin use was started before the onset of coronary heart disease. The number of coronary events among statin treated and untreated patients was divided by the total event-free survival. Event-free survival time was defined as the period from 1 January 1990 to the date of first coronary event, death, or censoring at the end of our observation.

We also compared survival among treated and untreated patients using the Kaplan-Meier survival method. In this analysis, we considered people to be untreated when they had not taken a statin for longer than one month before a coronary event or censoring. All patients who received a statin during follow-up for more than a month before an event or censoring were considered as treated.

We performed separate analyses for patients older than 55 years to compare them with the selected population from the Rotterdam study. Since the maximum follow-up in our cohort was 12 years, we chose 2002 as the end point in the Rotterdam study as well.

We used the Cox proportional hazard model to estimate the risk of coronary heart disease among statin treated patients compared with untreated patients. Since both groups had variable periods without statin treatment, we analysed statin treatment as a time dependent variable. This variable was equal to zero for the time statins were not used, and 1 for the time from start of statin treatment to the date of first coronary event or censoring. In the primary model, we adjusted the analyses for sex and year of birth.

In additional models we adjusted for other classic risk factors (see table 2). We performed subgroup analyses stratified by previously identified risk factors to define effect differences. For LDL cholesterol concentration, we used the median value to split the total population in two subpopulations. We classified high density lipoprotein (HDL) cholesterol concentration according to our normal laboratory values: $<0.9 \mathrm{mmol} / 1$ for males, $<1.1 \mathrm{mmol} / 1$ for females. To test for lifestyle improvements occurring at the start of statin treatment, we adjusted the Cox proportional hazard model for smoking cessation within six months after the start of statin treatment. We tested whether deselection of worst cases occurred by adding the patients who were never treated with statins to the treated group as if they had started treatment on 1 January 1990 and repeated the time dependent Cox proportional hazard analysis.

\section{RESULTS}

Of the 2400 patients recruited, we excluded 254 who already had coronary heart disease by 1990 . We excluded a further 188 patients because the type of lipid lowering treatment or the date of starting statin 
treatment was unknown, leaving 1950 patients with sufficient data on drug use (fig 1). In January 1990, 413 patients were treated with a statin, and a further 1294 patients were prescribed statins during follow-up. The mean delay in starting statin use was 4.3 years (SD 3.3 years). The 196 patients who were excluded because of missing data or loss to follow-up had serum LDL cholesterol concentrations $1.14 \mathrm{mmol} / \mathrm{l}$ higher than those of the included patients. We did not find other differences between these groups.

The patients who immediately received statin treatment in 1990 were on average 3.5 years older than the patients who started statin treatment later, had higher total and LDL cholesterol concentrations (both $\mathrm{P}<0.001)$, had lower HDL cholesterol levels $(\mathrm{P}=0.02)$, and were significantly more likely to be hypertensive than the other patients (table 1). Twenty eight patients stopped taking statins for unreported reasons.

The mean follow-up time was 8.5 years (SD 3.1 years). During 7473 person years of statin treated patients and 9319 person years of untreated patients, 408 patients had an incident coronary event, of whom 161 patients had been using statins for an average 3.4 years (median 2.7 years, range 1 month to 11.6 years). Most patients $(\mathrm{n}=1167)$ used simvastatin with a mean dose of $33 \mathrm{mg}$ (SD $20 \mathrm{mg}$ ), leading to $44 \%$ (SD 16\%) lower LDL cholesterol concentrations compared with before they started statin treatment. A further 211 patients used atorvastatin with a mean daily dose of $49 \mathrm{mg}$ providing a reduction in $\mathrm{LDL}$ cholesterol level of 49\% (SD 15\%). Less commonly used statins were pravastatin and fluvastatin. During statin treatment the mean total cholesterol concentration was $5.9 \mathrm{mmol} / \mathrm{l}$ (SD $1.2 \mathrm{mmol} / \mathrm{l})$, mean LDL cholesterol was $4.0 \mathrm{mmol} / \mathrm{l}$ (SD 1.2), and mean HDL cholesterol was $1.28 \mathrm{mmol} / \mathrm{l}(\mathrm{SD} 0.41)$.

In familial hypercholesterolaemia, the absolute risk of first onset of coronary heart disease was $11 / 1000$

Table 1 | General characteristics of patients with familial hypercholesterolaemia by their receipt of statin treatment in January 1990. Values are numbers (percentages) of patients unless stated otherwise

\begin{tabular}{|c|c|c|c|}
\hline & $\begin{array}{l}\text { Taking statins } \\
\quad(n=413)\end{array}$ & $\begin{array}{l}\text { Not taking statins } \\
\quad(n=1537)\end{array}$ & $\begin{array}{l}\text { P value of } \\
\text { difference }\end{array}$ \\
\hline Men & $197(48)$ & $727(47)$ & 0.89 \\
\hline Ever smoked & $281(77)$ & $1007(72)$ & 0.09 \\
\hline Hypertension & $47(11)$ & $48(3)$ & $<0.01$ \\
\hline Diabetes & $6(1)$ & $5(0.3)$ & 0.06 \\
\hline $\begin{array}{l}\text { Mean (SD) age at first visit to lipid clinic } \\
\text { (years) }\end{array}$ & $43.3(13.3)$ & $43.8(12.3)$ & 0.36 \\
\hline Mean (SD) age at 1 January 1990 (years) & $41.7(13.2)$ & $38.2(12.5)$ & $<0.001$ \\
\hline Mean (SD) body mass index $\left(\mathrm{kg} / \mathrm{m}^{2}\right)$ & $24.8(3.5)$ & $25.1(3.5)$ & 0.18 \\
\hline $\begin{array}{l}\text { Mean (SD) total cholesterol concentration } \\
(\mathrm{mmol} / \mathrm{l})\end{array}$ & $10.1(1.9)$ & $9.0(1.9)$ & $<0.001$ \\
\hline $\begin{array}{l}\text { Mean (SD) LDL cholesterol concentration } \\
(\mathrm{mmol} / \mathrm{l})\end{array}$ & $8.0(1.9)$ & $6.9(1.8)$ & $<0.001$ \\
\hline $\begin{array}{l}\text { Mean (SD) HDL cholesterol concentration } \\
(\mathrm{mmol} / \mathrm{l})\end{array}$ & $1.13(0.34)$ & $1.19(0.37)$ & 0.02 \\
\hline $\begin{array}{l}\text { Mean (SD) triglyceride concentration } \\
(\mathrm{mmol} / \mathrm{l})\end{array}$ & $1.45(0.71)$ & $1.58(0.48)$ & 0.007 \\
\hline
\end{tabular}

LDL=low density lipoprotein. HDL=high density lipoprotein.

All lipid concentrations are without statin treatment and adjusted for sex and year of birth.

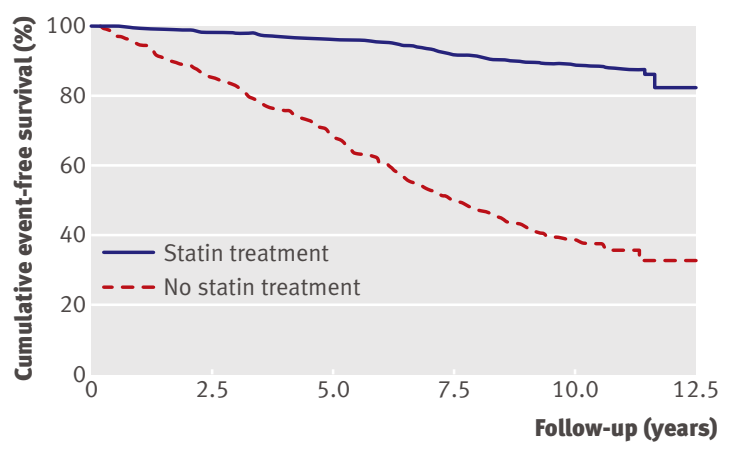

Fig 2 | Kaplan-Meier curve estimates of cumulative coronary heart disease-free survival among patients with familial hypercholesterolaemia according to statin treatment $(P<0.001$ for difference)

person years in statin treated patients compared with $119 / 1000$ person years in untreated patients. Incident coronary heart disease occurred at younger age in untreated patients (48.6 $v 50.9$ years, $\mathrm{P}=0.05$ ). The treated group had a significantly better event-free survival $(\mathrm{P}<0.001$, fig 2). After adjustment for year of birth and sex, statin treated patients had a $76 \%$ reduction in risk of coronary heart disease compared with untreated patients (hazard ratio $0.24(95 \%$ confidence interval 0.18 to 0.30$), \mathrm{P}<0.001)$. Further adjustment for baseline characteristics such as smoking, HDL and LDL cholesterol concentrations, diabetes, and hypertension resulted in an overall $82 \%$ risk reduction (hazard ratio $0.18(0.13$ to 0.25$), \mathrm{P}<0.001)$ (table 2).

As expected, men had a 2.5 times greater risk of coronary heart disease than women $(95 \%$ confidence interval 2.1 to $3.1, \mathrm{P}<0.001)$. Whether women with familial hypercholesterolaemia benefit from statins as men do is still under debate, ${ }^{14}$ and we therefore studied them separately. The women taking statins had a 79\% reduction in risk of coronary heart disease compared with women not taking statins (hazard ratio 0.21 (0.13 to 0.34$), \mathrm{P}<0.001)$; the men had an $83 \%$ risk reduction (hazard ratio 0.17 (0.11 to 0.26$), \mathrm{P}<0.001$ ) (table 2).

Patients who developed coronary heart disease had higher serum LDL cholesterol concentrations before treatment than did those without coronary heart disease $(7.5 \mathrm{mmol} / \mathrm{l}$ v $7.2 \mathrm{mmol} / \mathrm{l}, \mathrm{P}=0.03)$. During statin treatment, however, LDL cholesterol levels were identical among patients with and without coronary heart disease $(4.1 \mathrm{mmol} / 1$ v $4.0 \mathrm{mmol} / \mathrm{l}, \mathrm{P}=0.38)$. Classic risk factors were, as expected, more common in patients with coronary heart disease. The subgroup analyses in fig 3 suggest that the efficacy of statin treatment did not substantially differ between smokers and non-smokers, between patients with or without diabetes, and between patients with or without hypertension. However, the size of the subgroups were too small for meaningful analyses.

Of all 1288 patients who had ever smoked, 333 had stopped before 1990. A total of 407 statin users never smoked, and 388 had stopped before statin treatment was started. A further 105 patients $(10 \%$ of all those for 


\section{Subgroup}

Never smokers $(n=475)$ Ever smokers $(\mathrm{n}=1288)$

No diabetes $(n=1859)$

Diabetes $(n=90)$

No hypertension $(n=1722)$

Hypertension $(n=162)$

Serum LDL cholesterol $<7.0 \mathrm{mmol} / \mathrm{l}(\mathrm{n}=863)$

Serum LDL cholesterol $\geqslant 7.0 \mathrm{mmol} / \mathrm{l}(\mathrm{n}=788)$

HDL cholesterol low risk* $(n=1772)$

HDL high riskt $(n=162)$

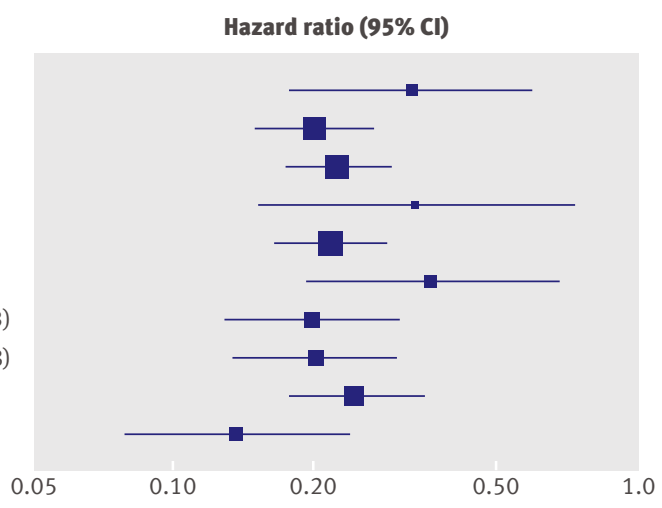

$\mathrm{LDL}=$ low density lipoprotein. $\mathrm{HDL}=$ high density lipoprotein

* Low risk serum concentrations: women $\geqslant 1.1 \mathrm{mmol} / \mathrm{l}$, men $\geqslant 0.9 \mathrm{mmol} / \mathrm{l}$

† High risk serum concentrations: women $<1.1 \mathrm{mmol} / \mathrm{l}$, men $<0.9 \mathrm{mmol} / \mathrm{l}$

Fig 3 | Subgroup analyses for reduction in risk of coronary heart disease in patients with familial hypercholesterolaemia by statin use in a time dependent analysis. Smoking, diabetes, and hypertension were compared based on having ever been present. LDL cholesterol concentration was split based on the median value, and HDL cholesterol level split based on normal laboratory values. Hazard ratio $(95 \% \mathrm{Cl})$ by statin use is shown on a logarithmic scale

whom smoking cessation date was known) quitted within six months of starting statin treatment. To test if lifestyle improvement related to the start of statin treatment could explain why smokers showed a larger risk reduction with statins, we adjusted for smoking cessation within six months of the start of statin treatment, but this did not materially change the effect of statin treatment on coronary heart disease risk (hazard ratio 0.20 (0.15 to 0.26$), \mathrm{P}<0.001$ ). Unfortunately, body mass index and waist circumference were not measured during follow-up.

A total of 243 patients in our cohort were never treated with statins. We performed an additional analysis adding those patients to the treatment group, as if statin treatment had started on 1 January 1990, to estimate the effect of an intention to treat analysis. The hazard ratio was even lower under these assumptions (data not shown).

We finally compared the risk of myocardial infarction in patients with familial hypercholesterolaemia who were older than 55 years $(n=261,64$ men) with that in 1975 people in a subgroup of the participants in the Rotterdam study. The mean age in both subgroups was 61.6 years and both had $24.5 \%$ men as a result of stratified selection from the Rotterdam study. The absolute risk of myocardial infarction was $6.7 / 1000$ person years in our statin treated patients, $60.5 / 1000$ person years in our untreated patients, and 4.1/1000 person years in the sample from the Rotterdam study. Event-free survival of our statin treated patients was not significantly different from that of the Rotterdam study sample (log rank test $\mathrm{P}=0.07$ ), whereas our untreated patients clearly had a higher risk of coronary heart disease $(\log$ rank test $\mathrm{P}<0.001)$ (fig 4). After adjustment for year of birth and sex, the point estimate of risk of myocardial infarction in our treated patients with familial hypercholesterolaemia was higher than the risk in the subgroup of the Rotterdam study, but this was not significant (hazard ratio 1.44 (0.80 to 2.60), $\mathrm{P}=0.23$ ), whereas the risk in our untreated patients was 8.7 times higher (hazard ratio 8.69 (4.77 to 15.82 ), $\mathrm{P}<0.001)$.

\section{DISCUSSION}

We report here that relatively modest doses of statins reduced the risk of coronary heart disease by about $80 \%$ in patients with familial hypercholesterolaemia. This is a much more pronounced reduction than was anticipated based on secular trends in earlier studies. ${ }^{78} \mathrm{We}$ also observed that statin treated patients older than 55 years had a risk of myocardial infarction approaching that of the general population. Finally, men and women experienced similar risk reductions in our study.

\section{Strengths and weaknesses of our study}

Our follow-up study has a number of limitations. Firstly, it was observational and not a randomised study. Therefore, the patients who started treatment immediately in 1990 may have represented a selected subgroup with more severe risk: they were selected first because they had further progressed in their disease process. If this were so, it would have resulted in a higher risk for the treated group compared with a randomised trial that distributed the risks equally. However, it seems unlikely that we have underestimated the risk reduction as the effect was unexpectedly large.

Secondly, it could be argued that our approach exaggerates the effect of the treatment, because our study was not placebo-controlled. Patients might have improved their lifestyle in conjunction with starting statin treatment. However, adjustment for smoking cessation within six months after the start of treatment (as a proxy for lifestyle improvement) did not change the effect of statin treatment.

Table 2 |Effect of statin treatment on risk of coronary heart disease in patients with familial hypercholesterolaemia by sex

\begin{tabular}{|c|c|c|c|c|c|c|c|c|}
\hline & \multicolumn{2}{|c|}{ Model I } & \multicolumn{2}{|c|}{ Model II } & \multicolumn{2}{|c|}{ Model III } & \multicolumn{2}{|c|}{ Model IV } \\
\hline & $\begin{array}{l}\text { Hazard ratio } \\
\qquad(95 \% \mathrm{Cl})\end{array}$ & $P$ value & $\begin{array}{l}\text { Hazard ratio } \\
(95 \% \mathrm{Cl})\end{array}$ & $P$ value & $\begin{array}{l}\text { Hazard ratio } \\
(95 \% \mathrm{Cl})\end{array}$ & $P$ value & $\begin{array}{l}\text { Hazard ratio } \\
(95 \% \mathrm{Cl})\end{array}$ & $P$ value \\
\hline All patients $(n=1950)$ & 0.24 (0.18 to 0.30$)$ & $<0.001$ & 0.22 (0.17 to 0.28$)$ & $<0.001$ & $0.20(0.15$ to 0.27$)$ & $<0.001$ & 0.18 (0.13 to 0.25$)$ & $<0.001$ \\
\hline Men $(n=924)$ & 0.20 (0.15 to 0.28$)$ & $<0.001$ & 0.20 (0.14 to 0.28$)$ & $<0.001$ & $0.16(0.11$ to 0.25$)$ & $<0.001$ & 0.17 (0.11 to 0.26$)$ & $<0.001$ \\
\hline Women $(n=1026)$ & $0.30(0.20$ to 0.43$)$ & $<0.001$ & 0.25 (0.17 to 0.37$)$ & $<0.001$ & 0.28 (0.18 to 0.43$)$ & $<0.001$ & 0.21 (0.13 to 0.34$)$ & $<0.001$ \\
\hline
\end{tabular}

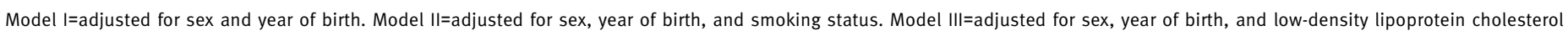

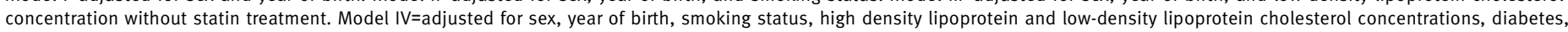
and hypertension. 


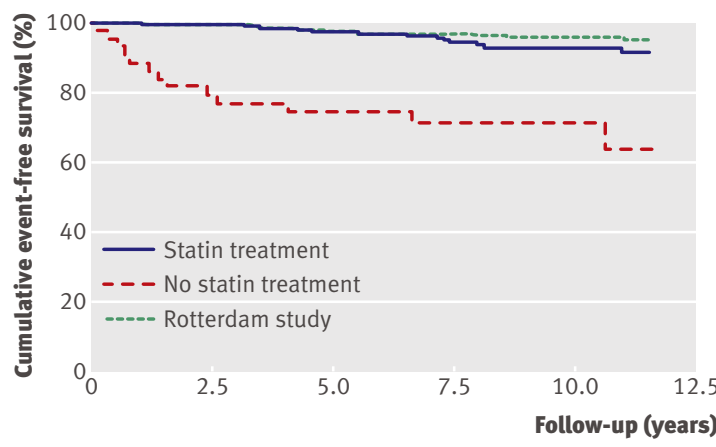

Fig 4 | Kaplan-Meier curve estimates of cumulative myocardial infarct-free survival among patients with familial hypercholesterolaemia older than $\mathbf{5 5}$ years according to statin treatment compared with a sample from the general population (Rotterdam study). ( $P<0.001$ for difference between untreated patients and general population; $\mathrm{P}=0.07$ for difference between treated patients and general population)

Thirdly, we analysed statin treatment as a time dependent variable, whereas an intention to treat analysis might have yielded smaller risk reductions. We analysed the 28 patients who stopped statin treatment as if they had stayed on treatment, in line with an intention to treat analysis. We also made an analysis in which all patients who were never treated with statins were added to the treated group as if they had started treatment on 1 January 1990, to estimate the effect that an intention to treat analysis could have had: this showed an even larger effect. Although this value is a rough estimation sensitive to choice of date of start of treatment, the decrease in hazard ratio indicates that our results are not overestimating the effect as a result of deselection of worst cases.

Although some of the weaknesses associated with lack of randomisation have been addressed, there is always the danger that unrecognised confounding factors, for which we made no adjustment, might have affected our results.

\section{Comparison with other studies}

The large risk reduction and the overlap of the eventfree survival between the treated patients and a sample of the general population (from Rotterdam study) suggest that statin treatment has profoundly improved the prognosis for familial hypercholesterolaemia.

Two previous studies have investigated this issue. A study in the United Kingdom compared mortality in patients with familial hypercholesterolaemia with that in the general population before and after the introduction of statins. ${ }^{7}$ Their results suggested that statins did indeed reduce mortality, but that mortality was still higher than in the general population. Exact information about the start date of statin treatment was not available, however, suggesting there might have been an unrecognised delay in statin initiation similar to what we found in the Netherlands.

In a much smaller study, 214 statin treated patients with familial hypercholesterolaemia still had increased risk of cardiovascular disease. ${ }^{8}$ As suggested by the high frequency of premature cardiovascular disease $(45 \%)$ in first degree relatives, the patients of this study might have been selected preferentially for severe risk of coronary heart disease. Moreover, statin treatment was not assessed against untreated familial hypercholesterolaemia and not in a time dependent fashion. The latter may have resulted in underestimation of the statin effect because of misclassification of periods without treatment. In contrast, in our study, we prospectively compared treated and untreated patients with familial hypercholesterolaemia as well as the general population in a direct manner and in the same calendar period.

\section{Implications of findings}

The standard treatment used currently is more aggressive than that used in our study: current regimens use simvastatin and atorvastatin doses up to $80 \mathrm{mg}$ daily. ${ }^{24}$ It should be emphasised, however, that in our study we excluded all prevalent cases, thereby restricting our study to primary prevention. Our results cannotbe extrapolated to secondary prevention, which may require more aggressive treatment.

Support for optimal treatment with statins can also be deduced from the results of the ENHANCE study: patients with familial hypercholesterolaemia had normal carotid intima-media thickness at baseline, and even simvastatin $80 \mathrm{mg}$ alone prevented thickening of the arterial wall. ${ }^{6}$

In previous studies we tested statin treatment of children with familial hypercholesterolaemia and showed attenuation of progression of carotid intimamedia thickness. ${ }^{1516}$ Our present study suggests that starting aggressive treatment during early childhood, as currently done and advised by the American Academy of Pediatrics, is probably not necessary to reduce coronary heart disease risk. ${ }^{17}$ Although atherosclerosis is present in children, this process is to a certain extent reversible, as shown by the close to normal intima-media thicknesses at baseline in the ENHANCE trial and our findings in the present study. In both studies, statin treatment was initiated after childhood. It is probably safe to limit statin treatment of children with heterozygous familial hypercholesterolaemia to those whose

\section{WHAT IS ALREADY KNOWN ON THIS TOPIC}

Statins are the first line treatment for familial

hypercholesterolaemia, but their efficacy is unknown because hard end points were not studied in placebo controlled trials

\section{WHAT THIS STUDY ADDS}

Lower statin doses than currently advised reduced coronary heart disease risk by $80 \%$ in patients with familial hypercholesterolaemia

Statin treated patients older than 55 years had a similar risk of myocardial infarction as did a sample from the general population of the same age 
first degree relatives have severe premature coronary heart disease.

In conclusion, our data show that lower statin doses than currently advised result in impressive reductions of coronary heart disease risk in patients with familial hypercholesterolaemia. These findings warrant an immediate start of statin treatment after familial hypercholesterolaemia has been diagnosed since such treatment leads to near normalisation of coronary heart disease risk.

Contributors: JV contributed to the original idea for the study and participated in the statistical analysis and writing the manuscript. DMO, MY, and JCW participated in the statistical analysis and writing the manuscript. JCD and DCGB participated in designing the study and collecting study data. AHL participated in collecting study data and writing the manuscript. JH participated in the statistical analysis and interpreting the data. JJPK initiated the large familial hypercholesterolaemia survey in 1989 in the Netherlands and holds the database of these patients. He also coached the research team and participated in writing of the manuscript. EJGS had the original idea for the study, coached the research team, and participated in writing the manuscript. EJGS is the guarantor of the study, and all authors read the article and approved the published version. Funding: This work was funded by the Netherlands Heart Foundation (2006B190). JJPK and EJGS have received research funding from pharmaceutical manufacturers, but they did not receive industry funding for the present study. All authors declare they are independent from funders.

Competing interests: None declared.

Ethical approval: The ethics institutional review board of each participating hospital approved the study protocol. All patients gave their informed consent.

Provenance and peer review: Not commissioned; externally peer reviewed.

1 Austin MA, Hutter CM, Zimmern RL, Humphries SE. Familia hypercholesterolemia and coronary heart disease: a HuGE association review. Am J Epidemiol 2004;160:421-9.

2 National Institute for Health and Clinical Excellence. Clinical guidelines and evidence review for familial hypercholesterolaemia: the identification and management of adults and children with familial hypercholesterolaemia. 2008 (Clinical guideline 71) www. nice.org.uk/CG71

3 Marks D, Thorogood M, Neil HA, Humphries SE. A review on the diagnosis, natural history, and treatment of familial hypercholesterolaemia. Atherosclerosis 2003;168:1-14.
4 Smilde TJ, van Wissen S, Wollersheim H, Trip MD, Kastelein JJ, Stalenhoef AF. Effect of aggressive versus conventional lipid lowering on atherosclerosis progression in familial hypercholesterolaemia (ASAP): a prospective, randomised, double-blind trial. Lancet 2001;357:577-81.

5 Van Wissen S, Smilde TJ, Trip MD, Stalenhoef AF, Kastelein JJ. Long term safety and efficacy of high-dose atorvastatin treatment in patients with familial hypercholesterolemia. Am J Cardiol 2005;95:264-6.

6 Kastelein JJ, Akdim F, Stroes ES, Zwinderman AH, Bots ML, Stalenhoef AF, et al. Simvastatin with or without ezetimibe in familial hypercholesterolemia. N Engl J Med 2008;358:1431-43.

7 Scientific Steering Committee on behalf of the Simon Broome Register Group. Mortality in treated heterozygous familial hypercholesterolaemia: implications for clinical management. Atherosclerosis 1999;142:105-12.

8 Mohrschladt MF, Westendorp RG, Gevers Leuven JA, Smelt AH. Cardiovascular disease and mortality in statin-treated patients with familial hypercholesterolemia. Atherosclerosis 2004;172:329-35.

9 Neil HA, Hammond T, Mant D, Humphries SE. Effect of statin treatment for familial hypercholesterolaemia on life insurance: results of consecutive surveys in 1990 and 2002. BMJ 2004;328:500-1.

10 Jansen AC, van Aalst-Cohen ES, Tanck MW, Trip MD, Lansberg PJ, Liem AH, et al. The contribution of classical risk factors to cardiovascular disease in familial hypercholesterolaemia: data in 2400 patients. J Intern Med 2004;256:482-90.

11 Jansen AC, van Aalst-Cohen ES, Hutten BA, Buller HR, Kastelein JJ, Prins MH. Guidelines were developed for data collection from medical records for use in retrospective analyses. J Clin Epidemiol 2005;58:269-74.

12 Friedewald WT, Levy RI, Fredrickson DS. Estimation of the concentration of low-density lipoprotein cholesterol in plasma, without use of the preparative ultracentrifuge. Clin Chem 1972;18:499-502.

13 Hofman A, Grobbee DE, de long PT, van den Ouweland FA Determinants of disease and disability in the elderly: the Rotterdam elderly study. Eur J Epidemiol 1991;7:403-22.

14 AbramsonJ, WrightJM. Are lipid-lowering guidelines evidence-based? Lancet 2007;369:168-9.

15 Wiegman A, Hutten BA, de GrootE, Rodenburg J, Bakker HD, Buller HR, et al. Efficacy and safety of statin therapy in children with familial hypercholesterolemia: a randomized controlled trial. JAMA 2004;292:331-7.

16 Rodenburg J, Vissers MN, Wiegman A, Van Trotsenburg AS, Van der Graaf A, De Goot E, et al. Statin treatment in children with familial hypercholesterolemia: the younger the better. Circulation 2007;116:664-8.

17 Daniels SR, Greer FR, and the Committee on Nutrition. Lipid screening and cardiovascular health in childhood. Pediatrics 2008;122:198-208.

Accepted: 26 October 2008 